\title{
3D imaging and stealth navigation instead of CT guidance for radiofrequency ablation of osteoid osteomas: a series of 52 patients
}

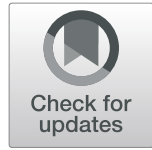

\author{
Ran Ankory ${ }^{1}$, Assaf Kadar ${ }^{1}$, Doron Netzer ${ }^{2}$, Haggai Schermann ${ }^{1 *}\left(\mathbb{D}\right.$, Yair Gortzak ${ }^{3}$, Shlomo Dadia ${ }^{3}$, \\ Yehuda Kollander ${ }^{3}$ and Ortal Segal ${ }^{3}$
}

\begin{abstract}
Background: Osteoid osteomas are benign bone neoplasms that may cause severe pain and limit function. They are commonly treated by radiofrequency ablation (RFA) through a needle inserted into the nidus of the lesion under CT guidance, which is associated with exposure of young patients to relatively high dose of radiation. The objective of this study was to investigate the amount of radiation, effectiveness and safety of an alternative imaging approach, the 3D image-guided (O-arm) technology and the Stealth navigation.
\end{abstract}

Methods: We retrospectively reviewed 52 electronic medical files of patients (mean age 24.7 years, range 8-59 years) who were treated with thermal ablation of benign osteoid osteomas guided by the navigated O-arm-assisted technique in our institution between 2015 and 2017. Data were extracted on the associated complications, the reduction in pain at 3 months and one year postoperatively, and the amount of radiation administered during the procedure.

Results: The level of pain on a visual analogue scale decreased from the preoperative average of 7.73 to 0 at the 3month follow-up. The mean dose-length product was $544.7 \mathrm{mGycm}{ }^{2}$ compared to the reported radiation exposure of 1971-7946 mGycm² of CT-guided radio ablations. The one intra-operative complication was a superficial burn in the subcutaneous lesion in a tibia that was treated locally with no major influence on recovery.

Conclusions: RFA ablation guided by 3D O-arm stealth navigation is as effective as the traditional CT-guided technique with the advantage of lower radiation exposure.

Trial registration: Retrospective study number 0388-17-TLV at Tel Aviv Sourasky Medical Center IRB, approved at 25.10.17.

Keywords: Osteoid osteoma, O-arm, Radiofrequency ablation, Radiation

\section{Background}

Osteoid osteomas are benign bone neoplasms that inflict severe pain. These lesions are small (usually between 10 and $20 \mathrm{~mm}$ in diameter) and are located mainly in the diaphysis of long bones. Osteoid osteomas account for 10$12 \%$ of all benign bone lesions and mostly affect patients between 5 to 24 years of age [1]. They are a self-limited condition, and are usually resolved without treatment within 1-7 years [1], but many patients require surgical intervention in order to decrease pain and regain function.

\footnotetext{
*Correspondence: sheralmi@bu.edu

'Division of Orthopedics, Tel Aviv Sourasky Medical Center affiliated with Tel

Aviv University, 153 Arlozorov st app 6, 6492211 Tel Aviv, Israel

Full list of author information is available at the end of the article
}

Until the late 1990's, the surgical treatment for osteoid osteomas consisted mainly of complete open resection [2]. This treatment was very effective but had several disadvantages. The tumor is difficult to locate and identify under direct vision, thus posing the risks of incomplete resection and tumor recurrence [3]. Additionally, resection of a tumor in a weight-bearing bone might require restrictions of activities or even prophylactic fixation.

The current mainstay of treatment for osteoid osteomas is computerized tomographic (CT)-guided radiofrequency ablation (RFA). This is a minimally invasive procedure with excellent clinical results [4-6]. Complications are rare and may include nerve irritation or superficial burns that are usually resolved without intervention. Rosenthal et al.

(c) The Author(s). 2019 Open Access This article is distributed under the terms of the Creative Commons Attribution 4.0 International License (http://creativecommons.org/licenses/by/4.0/), which permits unrestricted use, distribution, and reproduction in any medium, provided you give appropriate credit to the original author(s) and the source, provide a link to the Creative Commons license, and indicate if changes were made. The Creative Commons Public Domain Dedication waiver (http://creativecommons.org/publicdomain/zero/1.0/) applies to the data made available in this article, unless otherwise stated. 
reported that CT-guided RFA lowered the hospital stay from an average of 4.7 days to 0.18 days [7]. The downside of this procedure is the significant levels of emission of ionizing radiation from the repeated CT scans. High levels of radiation emissions are of special concern when treating osteoid osteomas since they are common among children and young adults and might put them at risk for future radiation-related complications [8].

Several authors have recently described the benefit of using three-dimensional (3D) intra-operative images with an O-arm in combination with a navigation system. They were able to accurately direct implants into demanding anatomical locations without radiation exposure [9-13]. Most of the literature on $\mathrm{O}$-arm-assisted navigation systems is based on spine surgery procedures in which multiple implants (e.g., pedicular screws) are inserted with maximal accuracy and minimal ionizing radiation. The goals of this study were, therefore, to describe a new technique that utilizes a navigation system combined with an $\mathrm{O}$-arm that can help direct the surgeon into bony lesions with minimal radiation to the patient and staff compared to conventional CT-guided procedures. Specifically, we asked: is RFA of osteoid osteomas using 3D image-guided (O-arm) technology and the StealthStation navigation system effective? (2) Does RFA using 3D image-guided (O-arm) technology reduce the amount of radiation exposure compared to CTguided radio ablations? (3) Is radio frequency ablation using 3D image-guided (O-arm) technology safe?

\section{Methods}

This study was approved by our institutional review board on November 2017 (705-17-TLV). Medical records were reviewed for demographic and clinical data, primary tumor type and anatomical location. Outcome measures were pain scores (utilizing the clinically implemented visual analogue scale) and complications following the procedure. Radiation exposure was quantified by dose-length product (DLP), which is a measurement calculated by the scan length, and the volume CT dose index (CTI). DLP is easily acquired, reproducible, and conforms to regulatory standards $[14,15]$. Data were collected using the institutional PACS system by reviewing intraoperative images, dose reports, and interpretative reports.

Between 2015 and 2017, we used the O-arm StealthStation navigation system to treat 52 patients for osteoid osteomas. All 52 patients were available for follow-up for a minimum of 36 months (range, 36-60 months). They included 31 males and 21 females whose mean age at the time of surgery was 24.7 years (range, $8-59$ years). None of the patients had received prior surgical treatment for the index lesions. All the patients were diagnosed with osteoid osteomas based on clinical findings and imaging studies. Biopsies of 12 patients were retrieved during the procedure and the specimens were confirmed as osteoid osteomas by a musculoskeletal pathologist. Most of the lesions were located at the femur, while other sites included the tibia, the forearm, and the pelvis (Table 1). Patients with a preoperative pain score of 5 on a visual analogue scale (VAS) were excluded from the study.

The surgical procedure was carried out in the operating room with the patient under regional or general anesthesia. The patient was placed on a radiolucent, fluoroscopy-capable operating table, and the affected extremity was prepared and draped in a sterile manner. The optical tracking array was secured to the patient either by silk sutures to the skin in proximity to the lesion or by bony anchor devices, depending on the anatomical location of the lesion. (Fig. 1a). The patient was then scanned by a cone beam CT $\left(\mathrm{O}-\mathrm{arm}^{\circ}\right.$ scanner Medtronic Sofamor, Danek Memphis, TN, USA) to locate the lesion. A 3D image was created by the navigation system (StealthStation $^{\circ}$, Medtronic Sofamor, Danek Memphis, TN, USA) and by a set of calibrated navigational instruments (mainly a drill bit or a Jamshidi needle) (Fig. 1b), and the bone and the lesion were penetrated using real-time navigation (Fig. 2). The lesion was then curetted and specimens were sent for biopsy. A 15-cm RF probe needle (RITA Angiodynamics Inc., USA or Covidien Ltd. USA, cool tip 15-cm long) was then advanced without any additional fluoroscopy images or scans. When the needle came in contact with the lesion, a second scan was performed to verify the location of the needle tip, after which the ablation was performed according to a protocol of $90^{\circ} \mathrm{C}$ for $6-9 \mathrm{~min}$ (Fig. 1c). Lastly, the skin was sutured with 3-0 nylon sutures, and the patient's skin was examined for burns or other superficial complications. The postoperative protocol included immediate weight bearing.

Statistical analysis Means and ranges were used to describe continuous variables, and categorical variables are presented as numbers (percentages). Univariate analyses were performed with the Student's t-test for continuous variables. The level of significance was set at $p=0.05$.

\begin{tabular}{ll}
\multicolumn{1}{l}{ Table 1 Patient demographic and medical data $(n=52)$} \\
\hline Parameter & Values \\
\hline Male sex & $31(60 \%)$ \\
Mean age, years & 24.7 (range $8-59)$ \\
Lesion location & \\
Femur & $25(48 \%)$ \\
Tibia & $11(21 \%)$ \\
Radius ulna & $5(9.6 \%)$ \\
Pelvis & $4(7.7 \%)$ \\
Other & $7(13.5 \%)$ \\
Preoperative VAS for pain & $7.73($ range $5-10)$
\end{tabular}

VAS visual analog scale 


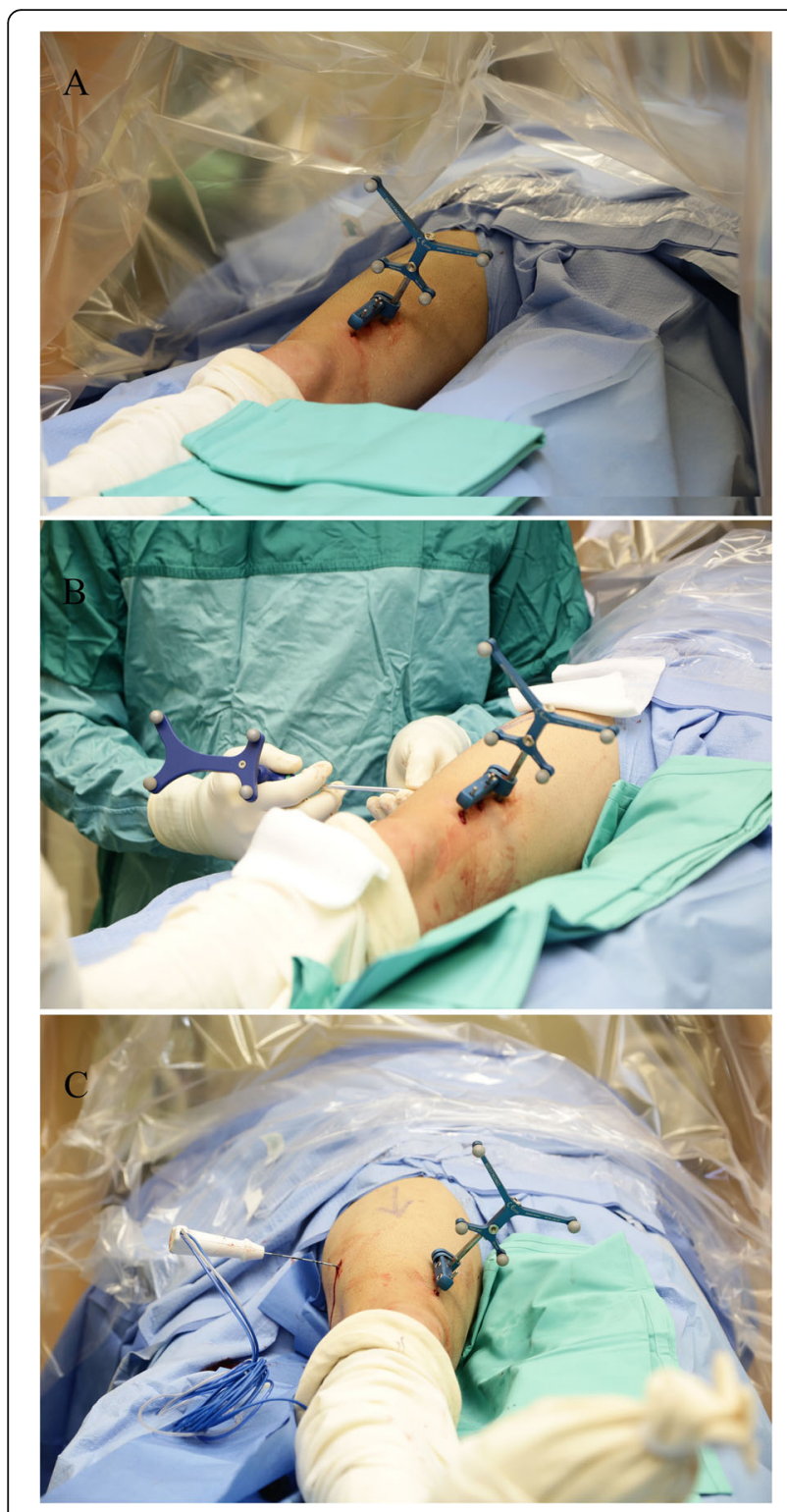

Fig. 1 A 20-30 year old patient with right femoral osteoid osteoma treated with radiofrequency ablation guided by three-dimensional (3D) image-guided (O-arm) technology. a The Optical tracking frame is fixed to the femur and then calibrated to the navigation system. $\mathbf{b}$ A navigated Jamshidi needle is introduced to the bone and penetrating the lesion with real time navigation. c A Radiofrequency probe needle is inserted into the lesion, followed by ablation according to a protocol of $90^{\circ} \mathrm{C}$ for $6-9$ min

\section{Results}

The average duration of the entire procedure was 65 min (range $55-100 \mathrm{~min}$ ), with an average radio ablation time of $7 \mathrm{~min}(6-9 \mathrm{~min})$. The navigation system helped place the RF needle accurately into the lesion in all cases, as verified in repeat scans prior to the ablation. All the patients were discharged one day after surgery.

All but one of the 52 patients remained asymptomatic at the 3-week and 3-month follow-up visits. Their pain scores decreased from a preoperative average of 7.73 (range 5-9) to 0.5 (range 0-1) at the first follow-up visit at 3 weeks $(p=0.03)$. Three $(5.7 \%)$ patients had pain recurrence and underwent repeated ablations within the first year postoperatively. The repeated ablation provided a definitive solution for in all cases. No lesion recurrences were noted at the 36- to 60-month follow-up.

The mean DLP was $544.7 \mathrm{mGycm}^{2}$ (range 180-2959). The variations in DLP were due to the requirement of repeated scans at various anatomical locations in order to ensure the correct placement of the needle tip.

There was one intra-operative complication of a superficial burn in a patient who had a lesion in the tibia. The burn was due to the lesion's location in a relatively subcutaneous part of the tibia, and it was treated locally without any major sequelae.

\section{Discussion}

The questions we addressed in this retrospective analysis were the effectiveness and safety of the RFA of osteoid osteomas using 3D image-guided (O-arm) technology and StealthSystem navigation for the treatment of osteoid osteomas. Our findings demonstrated that it had provided excellent clinical results in our cohort of $52 \mathrm{pa}-$ tients, with significant pain relief, and mild operative complications. Notably, radiation exposure was reduced compared to the traditional method of inserting the RFA needle under CT guidance.

Osteoid osteomas frequently require surgical treatment for pain relief. While open surgical resection and curettage is an excellent option, it is extensive surgery with the risk of side effects, including a $4.5 \%$ fracture rate [2]. Minimal invasive RFA is an acceptable alternative to open surgery. Many studies have shown that RFA provides excellent clinical results with minimal risk and complications $[3,16]$. Various imaging modalities have been introduced in recent years in order to guide the RF needle precisely into the lesion's nidus. The most common modality is standard CT, and the others include ultrasonography, fluoroscopy and magnetic resonance imaging $[4,5,17]$. Lindner at el. presented their results of 58 CT-guided ablations with a $95 \%$ success rate after the first ablation attempt and a 100\% success rate after a second ablation [17]. Rimondi et al. considered that the key for obtaining a good result is complete removal of the lesion, thus stressing the importance of accurate guidance of the needle into the nidus [18].

$3 \mathrm{D}$ navigation provides a multi-planar view that allows the surgeon the freedom to find the safest tract to the lesion in real-time navigation without additional radiation exposure. Another advantage of the $\mathrm{O}$-arm approach is the ability to confirm the accurate position of the RF needle. The amount of radiation exposure during traditional CT-guided RFA varies considerably in the literature. Leng 


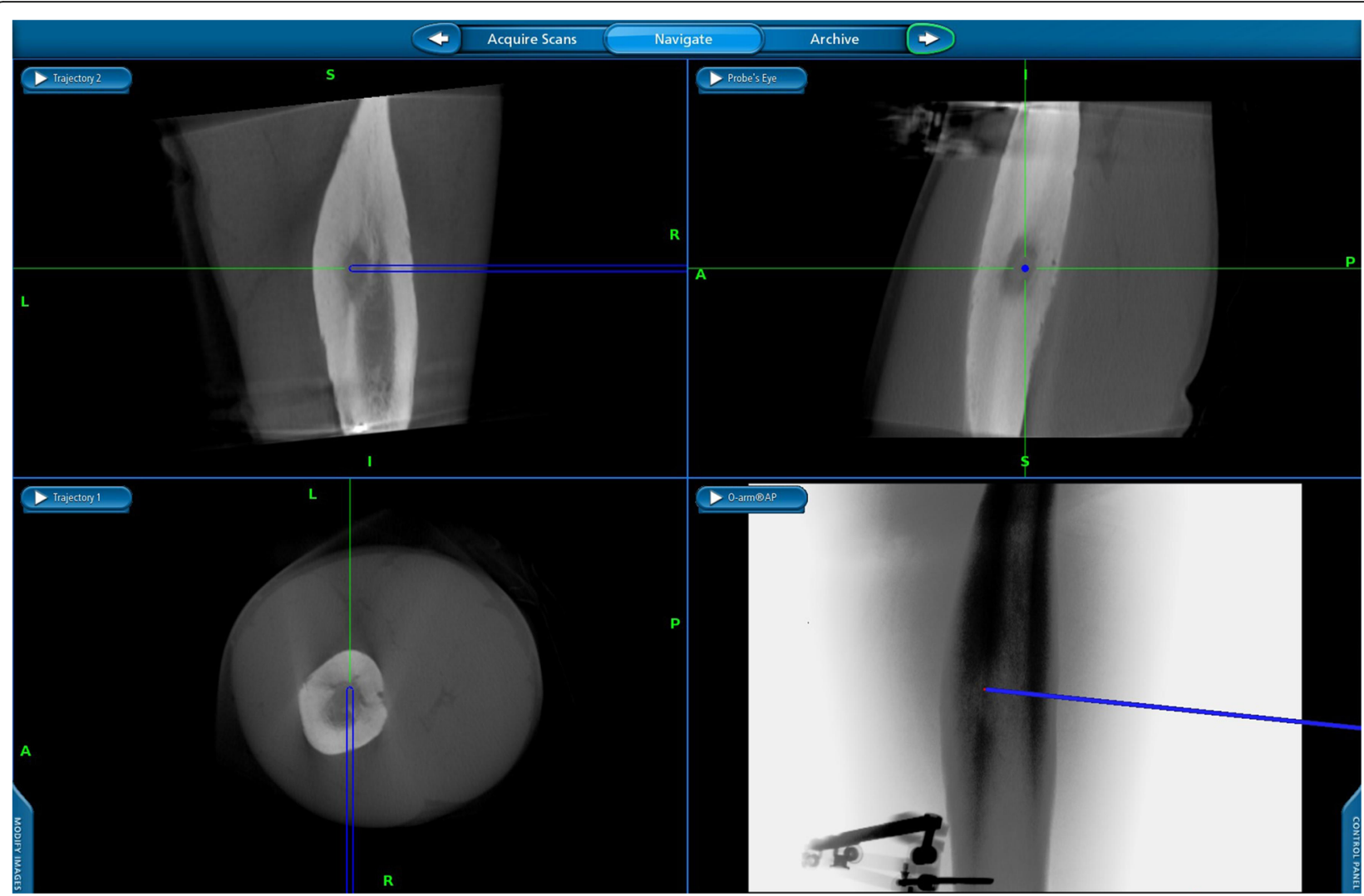

Fig. 2 Real time navigation image (O-Arm) of a Jamshidi needle introduced into the osteoid osteoma. The figure presents coronal (upper right panel), sagittal (upper left panel), axial (lower right panel) and 3 dimensional (lower left panel) reconstructions

et al. reported their experience in 42 ablations and found high levels of radiation exposure (a mean DLP of $7946 \pm$ $3351 \mathrm{mGyCm}^{2}$ ) [19]. Tsalafoutas et al. reported their experience in 14 RFA procedures and measured a mean DLP of $1971 \mathrm{mGycm}^{2}$ (range 994-3232) [20]. These large variations in radiation exposure can be explained by the different anatomical sites of the lesion and the surgeon's skill in locating it. The mean DLP of $544.7 \mathrm{mGycm}^{2}$ (range 180-2959 $\mathrm{mGycm}^{2}$ ) in our current report was considerably lower. For convenience, previously reported data regarding DLP of ablations with conventional CT vs Oarm fluoroscopy is summarized in Table 2. Another advantage of the $\mathrm{O}$-arm and stealth navigation is that it is carried out in the operating room, thus enabling a switch to general anesthesia in cases where local anesthesia fails, and as well as transfer to open surgery when required.

Our results correlate with those of Cheng at el's report on the advantages of the $\mathrm{O}$-arm and navigation system. In their comparative study of 66 patients, excellent pain relief was achieved both with conventional CT-guided RFA and with the O-arm navigated RFA. Notably, those authors also noted a significant two-fold reduction in radiation exposure in the $\mathrm{O}$-arm group [21].

Perry et al. have compared use of cone-beam computed tomography (CBCT) with two-axis fluoroscopic navigational overlay and conventional $\mathrm{CT}$ guidance for

Table 2 Previously reported data comparing O-arm navigation and CT guidance for osteoid osteoma ablation

\begin{tabular}{|c|c|c|c|c|}
\hline Study & Type of guidance & Number of patients & Average ED (mSv) & Average DLP (mGy-cm²) \\
\hline This study (Segal et al) & O-arm with navigation & 52 & - & 544.7 \\
\hline \multirow[t]{2}{*}{ Cheng et al., 2014} & O-arm with navigation & 23 & - & 446.6 \\
\hline & Conventional CT guidance & 36 & - & 1058.8 \\
\hline Leng et al., 2011 & Conventional CT guidance & 42 & 119.7 & 7946 \\
\hline Tsalafoutas et al., 2007 & Conventional CT guidance & 14 & 35 & 1976 \\
\hline Perry et al., 2017 & Conventional CT guidance & 55 & 39 & 615 \\
\hline
\end{tabular}


osteoid osteoma ablation in a pediatric cohort. Interestingly, CBCT was associated with more than three-fold reduction in radiation dose (0.12 vs. $0.39 \mathrm{mSv}, p=0.02)$ [22]. These results urge direct comparison between $\mathrm{CBCT}$ and $\mathrm{O}$-arm navigation.

Surgical navigation techniques are not without drawbacks. They require an expensive setup and skilled personnel, and intraoperative CT and navigation systems are available in a very limited number of hospitals. The adverse effects of surgical navigations include fractures around the reference frame pin, which was reported in up to $1 \%$ of navigated total knee replacements [23].

Several limitations of this study bear mention, It is a retrospective analysis that was carried out on a small group of patients in the absence of a control group. The variation in the levels of radiation is high and could not be accurately compared to radiation exposure in CTguided RFA without a control group of similar lesion location and number of patients.

\section{Conclusions}

We found that intraoperative O-arm technology in combination with 3D StealthSystem navigation is a safe and effective technique in the treatment of osteoid osteomas and should be considered as an alternative to conventional CT-guided interventions with the aim of reducing radiation exposure, especially among younger patients. Additional research is required to compare $\mathrm{O}$-arm with novel cone-beam CT.

\section{Acknowledgements}

None.

\section{Authors' contributions}

RA - study design, data accumulation and analysis, manuscript drafting, final approval of the manuscript. AK - study design, data interpretation, manuscript drafting, final approval of the manuscript. HS - data analysis, critical review of the manuscript, final approval of the manuscript. DN study design, critical review of the manuscript, final approval of the manuscript. YG - data accumulation and analysis, critical review of the manuscript, final approval of the manuscript. SD - study design, manuscript drafting, final approval of the manuscript. YK - data interpretation, critical review of the manuscript, final approval of the manuscript. OS - study design, manuscript drafting, final approval of the manuscript.

\section{Funding}

The authors received no funding.

\section{Availability of data and materials}

The datasets used and/or analysed during the current study are available from the corresponding author on reasonable request.

\section{Ethics approval}

Retrospective study number 0388-17-TLV at Tel Aviv Sourasky Medical Center Institutional Review Board, approved at 25.10.17.

\section{Consent for publication}

The requirement for consent for publication by the study subjects was waived by the Tel Aviv Sourasky Medical Center Institutional Review Board, as a retrospective study of existing data.

\section{Competing interests}

The authors declare that they have no competing interests.

\section{Author details}

${ }^{1}$ Division of Orthopedics, Tel Aviv Sourasky Medical Center affiliated with Tel Aviv University, 153 Arlozorov st app 6, 6492211 Tel Aviv, Israel. ${ }^{2}$ Meir Medical Center, Kfar Sava, Israel affiliated with Tel Aviv University, Tel Aviv, Israel. ${ }^{3}$ The National Unit for Orthopedic Oncology, Tel Aviv Sourasky Medical Center affiliated with Tel Aviv University, Tel Aviv, Israel.

Received: 14 April 2019 Accepted: 22 November 2019

Published online: 01 December 2019

\section{References}

1. Greenspan A. Benign bone-forming lesions: osteoma, osteoid osteoma, and osteoblastoma. Clinical, imaging, pathologic, and differential considerations. Skelet Radiol. 1993;22:485-500. http://www.ncbi.nlm.nih.gov/pubmed/8272884

2. Campanacci M, Ruggieri P, Gasbarrini A, Ferraro A, Campanacci L. Osteoid osteoma. Direct visual identification and intralesional excision of the nidus with minimal removal of bone. J Bone Joint Surg Br. 1999;81:814-20. http:// www.ncbi.nlm.nih.gov/pubmed/10530842

3. Atesok Kl, Alman BA, Schemitsch EH, Peyser A, Mankin H. Osteoid osteoma and osteoblastoma. J Am Acad Orthop Surg. 2011;19:678-89. http://www. ncbi.nlm.nih.gov/pubmed/22052644.

4. Barei DP, Moreau G, Scarborough MT, Neel MD. Percutaneous radiofrequency ablation of osteoid osteoma. Clin Orthop Relat Res. 2000: 115-24. http://www.ncbi.nlm.nih.gov/pubmed/10810468

5. Marić D, Djan I, Petković L, Vidosavljević M, Sopta J, Marić D, et al. Osteoid osteoma. J Pediatr Orthop B. 2011;20:46-9. https://doi.org/10.1097/BPB. Ob013e32833fb8bc.

6. Neumann D, Berka H, Dorn U, Neureiter D, Thaler C. Follow-up of thirtythree computed-tomography-guided percutaneous radiofrequency thermoablations of osteoid osteoma. Int Orthop. 2012;36:811-5. https://doi. org/10.1007/s00264-011-1402-8.

7. Rosenthal DI, Hornicek FJ, Wolfe MW, Jennings LC, Gebhardt MC, Mankin $\mathrm{HJ}$. Percutaneous radiofrequency coagulation of osteoid osteoma compared with operative treatment. J Bone Joint Surg Am. 1998;80:815-21. http:// www.ncbi.nlm.nih.gov/pubmed/9655099.

8. Brody AS, Frush DP, Huda W, Brent RL. American Academy of Pediatrics section on radiology. Radiation risk to children from computed tomography. Pediatrics. 2007;120:677-82. https://doi.org/10.1542/peds.2007-1910.

9. Costa F, Ortolina A, Attuati L, Cardia A, Tomei M, Riva M, et al. Management of C1-2 traumatic fractures using an intraoperative 3D imaging-based navigation system. J Neurosurg Spine. 2015:128-33. https://doi.org/10.3171/ 2014.10.SPINE14122.

10. Geiger D, Napoli A, Conchiglia A, Gregori LM, Arrigoni F, Bazzocchi A, et al. MR-guided focused ultrasound (MRgFUS) ablation for the treatment of nonspinal osteoid osteoma. J Bone Jt Surgery-American. 2014;96:743-51. https://doi.org/10.2106/JBJS.M.00903.

11. Kassis SZ, Abukwedar LK, Msaddi AK, Majer CN, Othman W. Combining pedicle screw stimulation with spinal navigation, a protocol to maximize the safety of neural elements and minimize radiation exposure in thoracolumbar spine instrumentation. Eur Spine J. 2016;25:1724-8. https:// doi.org/10.1007/s00586-015-3973-3.

12. Luo TD, Polly DW, Ledonio CG, Wetjen NM, Larson AN. Accuracy of pedicle screw placement in children 10 years or younger using navigation and intraoperative CT. Clin Spine Surg. 2016;29:E135-8. https://doi.org/10.1097/ BSD.0000000000000230.

13. Suárez-Huerta M, Roces-Fernández A, Mencía-Barrio R, Alonso-Barrio JA, Ramos-Pascua LR. Periprosthetic femoral fractures after hemiarthroplasty. An analysis of 17 cases. Rev Esp Cir Ortop Traumatol. 2015;59:333-42. https:// doi.org/10.1016/j.recot.2014.11.002.

14. Huda W, Mettler FA. Volume CT dose index and dose-length product displayed during CT: what good are they? Radiology. 2011;258:236-42. https://doi.org/10.1148/radiol.10100297.

15. Tsalafoutas IA, Metallidis SI. A method for calculating the dose length product from CT DICOM images. Br J Radiol. 2011;84:236-43. https://doi.org/ 10.1259/bjr/37630380.

16. Cantwell CP, Obyrne J, Eustace S. Current trends in treatment of osteoid osteoma with an emphasis on radiofrequency ablation. Eur Radiol. 2004;14: 607-17. https://doi.org/10.1007/s00330-003-2171-6. 
17. Lindner NJ, Ozaki T, Roedl R, Gosheger G, Winkelmann W, Wörtler K. Percutaneous radiofrequency ablation in osteoid osteoma. J Bone Joint Surg Br. 2001;83:391-6. http://www.ncbi.nlm.nih.gov/pubmed/11341426

18. Rimondi E, Bianchi G, Malaguti MC, Ciminari R, Del Baldo A, Mercuri M, et al. Radiofrequency thermoablation of primary non-spinal osteoid osteoma: optimization of the procedure. Eur Radiol. 2005;15:1393-9. https://doi.org/ 10.1007/s00330-004-2616-6.

19. Leng S, Christner JA, Carlson SK, Jacobsen M, Vrieze TJ, Atwell TD, et al. Radiation dose levels for interventional CT procedures. Am J Roentgenol. 2011;197:W97-103. https://doi.org/10.2214/AJR.10.5057.

20. Tsalafoutas IA, Tsapaki V, Triantopoulou C, Gorantonaki A, Papailiou J. CTguided interventional procedures without CT fluoroscopy assistance: patient effective dose and absorbed dose considerations. Am J Roentgenol. 2007; 188:1479-84. https://doi.org/10.2214/AJR.06.0705.

21. Cheng EY, Naranje SM, Ritenour ER. Radiation dosimetry of intraoperative cone-beam compared with conventional CT for radiofrequency ablation of osteoid osteoma. J Bone Joint Surg Am. 2014;96:735-42. https://doi.org/10. 2106/JBJS.M.00874.

22. Perry BC, Monroe EJ, McKay T, Kanal KM, Shivaram G. Pediatric percutaneous osteoid osteoma ablation: cone-beam CT with fluoroscopic overlay versus conventional CT guidance. Cardiovasc Intervent Radiol. 2017 Oct;40(10): 1593-9. https://doi.org/10.1007/s00270-017-1685-2 Epub 2017 May 11.

23. Bauwens K, Matthes G, Wich M, Gebhard F, Hanson B, Ekkernkamp A, et al. Navigated total knee replacement. A meta-analysis. J Bone Joint Surg Am. 2007;89:261-9. https://doi.org/10.2106/JBJS.F.00601.

\section{Publisher's Note}

Springer Nature remains neutral with regard to jurisdictional claims in published maps and institutional affiliations.

Ready to submit your research? Choose BMC and benefit from:

- fast, convenient online submission

- thorough peer review by experienced researchers in your field

- rapid publication on acceptance

- support for research data, including large and complex data types

- gold Open Access which fosters wider collaboration and increased citations

- maximum visibility for your research: over $100 \mathrm{M}$ website views per year

At $\mathrm{BMC}$, research is always in progress.

Learn more biomedcentral.com/submissions 